\title{
Hábito y calidad del desayuno en alumnos de dos escuelas primarias públicas de la ciudad de Santa Fe
}

\author{
Breakfast habit and quality in students from two public \\ primary schools in the city of Santa Fe
}

\author{
Lic. en Nutrición Valeria Fugasa, Lic. en Nutrición Eugenia Bertaa, \\ Msc. Florencia Walz ${ }^{b}$ Dra. Ma. Alejandra Fortino y Dra. Marcela I. Martinelli
}

\section{RESUMEN}

Introducción. El desayuno proporciona la energía y los nutrientes necesarios que permiten un óptimo rendimiento físico e intelectual. Objetivo. Conocer la frecuencia del desayuno, los factores que condicionan el hábito y su omisión, la calidad del desayuno y la colación en los niños de escuelas públicas de la ciudad de Santa Fe. Población y métodos. Estudio descriptivo transversal. Se evaluaron 637 alumnos mediante una encuesta estructurada de respuestas cerradas.

Resultados El 75\% de los niños desayunaban diariamente; solo $1,6 \%$ realizaban un desayuno de buena calidad. Consumir el desayuno todos los días o a veces no dependía de la compañía de un familiar. De $1^{\circ}$ a $5^{\circ}$ grado la frecuencia semanal dependió de que algún familiar lo preparara $(p=0,04)$. La causa principal de omisión del desayuno fue la falta de tiempo. El 23\% de los alumnos de $6^{\circ}-7^{\circ}$ grado manifestaron sentir malestar al desayunar. Más del $50 \%$ de los niños miraban televisión mientras desayunaban.

a. Departamento de Ciencias Biológicas.

b. Departamento de Matemática y Estadística Aplicada. Facultad de Bioquímica y Ciencias Biológicas. Universidad Nacional del Litoral, Santa Fe, Argentina.

Correspondencia: Dra. Marcela Martinelli: mmartine@fbcb.unl. edu.ar

Conflicto de intereses: Ninguno que declarar.

Financiación: Universidad Nacional del Litoral. Proyecto de Extensión de Interés Social. UNL N ${ }^{\circ} 500 / 11$. Expte. $\mathrm{N}^{\circ} 571334$.

Recibido: 21-2-2013 Aceptado 3-6-2013
La mayoría de los alumnos de $1^{\circ}$ a $3^{\text {er }}$ grado consumieron un desayuno de mejorable $(41 \%) \mathrm{e}$ insuficiente calidad (41\%). En los alumnos de $4^{\circ}$ y $5^{\circ}$ grado predominó el de insuficiente calidad $(50 \%)$. En el grupo de $6^{\circ}$ y $7^{\circ}$ grado fue notable el consumo de desayuno de mala calidad (16\%). En muy pocos niños la colación resultó apropiada para completar la calidad final del desayuno. Conclusiones. Si bien se encontró un alto porcentaje de escolares que tienen el hábito de desayunar, la mayoría lo hacen de forma incompleta. Muy pocos logran completar la calidad del desayuno con los alimentos ingeridos a media mañana.

Palabras clave: frecuencia de desayuno, calidad del desayuno, colación, escolares.

http:/ /dx.doi.org/10.5546/aap.2013.502

\section{INTRODUCCIÓN}

Durante la edad escolar, la nutrición adecuada desempeña un papel fundamental para que el niño alcance su pleno potencial de crecimiento, desarrollo y salud. El desayuno tiene gran importancia, ya que proporciona la energía y los nutrientes necesarios para comenzar el día, lo que permite un óptimo rendimiento físico e intelectual..$^{1-4}$

Numerosos estudios han observado que los niños que no desayunan no cubren la ingesta diaria recomendada de vitaminas $\mathrm{A}$, E, B6 y folato, y de minerales como calcio, hierro y cinc. ${ }^{5-7}$ A pesar de la importancia del desayuno, su omisión o realización incorrecta o insuficiente es una conducta observada con frecuencia en los niños de numerosos países. $^{8-11}$

Los estudios realizados en España con 5000 niños y jóvenes de 2 a 25 años indicaron que $8 \%$ a $9 \%$ no desayunaban y solo $25 \%$ a $29 \%$ realizaban un desayuno adecuado. ${ }^{12}$ Un estudio realizado en escolares del Gran Buenos Aires, de 7 a 12 años, reveló que $94 \%$ desayunaban, pero solo $5 \%$ realizaban un desayuno de calidad nutricional completa. ${ }^{13}$ Las razones alegadas para la omisión fueron diversas, destacando: "no me apetece", "me sienta mal", "estoy cansado", "no tengo tiempo", "tengo que prepararlo yo mismo" o "tengo que desayunar solo". ${ }^{13,14}$

Un desayuno de calidad nutricional completa y balanceada incluye al menos una porción de alimento de tres grupos: lácteos, cereales y frutas. ${ }^{1,15-17}$ Las Guías Alimentarias para la Población Argentina destacan la importancia de comenzar el día con un buen desayuno al levantarse y completarlo 
durante la mañana con una colación. La principal función de este refrigerio debería ser completar la calidad del desayuno. ${ }^{16}$

La escasez de datos a nivel nacional y local respecto del consumo de desayuno en los escolares motivó la realización de este estudio, cuyos objetivos fueron:

- Conocer la frecuencia semanal de consumo de desayuno y factores asociados al hábito de desayunar en escolares de dos escuelas públicas de la ciudad de Santa Fe.

- Determinar la calidad del desayuno y la calidad final luego de la colación.

\section{POBLACIÓN Y MÉTODOS}

El estudio se realizó en el período abrilmayo de 2012, en dos de las cuatro escuelas primarias públicas de la zona centro de la ciudad de Santa Fe teniendo en cuenta que ambas coincidían en: no recibir asistencia alimentaria, disponer de kiosco escolar, presentar un nivel socioeconómico similar de los alumnos y tener matrículas semejantes. Fueron seleccionadas por la Federación de Asociaciones de Cooperadoras Escolares según la predisposición de los equipos directivos para llevar a cabo la investigación.

La matrícula total entre ambas escuelas fue de 817 alumnos en el turno mañana. Se realizó un estudio descriptivo transversal con los alumnos de $1^{\circ}$ a $7^{\circ}$ grado, que aceptaron participar voluntariamente y cuyos padres otorgaron el consentimiento informado por escrito. Se excluyeron del análisis quienes declararon haber modificado sus patrones alimentarios como consecuencia de alguna enfermedad preexistente. Se llegó así a una muestra constituida por 637 alumnos de ambos sexos.

Se utilizó una encuesta basada en la empleada por Angeleri y cols. El instrumento fue modificado con opciones de respuestas cerradas para los motivos de no desayunar y se agregaron preguntas que permitieron definir los factores que se asociaban a la frecuencia de desayuno de interés en este estudio. Además, se consideró la cantidad ingerida de cada alimento en el desayuno y no solo la variedad, y se excluyó a los niños que no desayunaban de la categoría mala calidad definida por la autora.

Las encuestas fueron realizadas en forma individual por 10 encuestadores entrenados (tesinistas y licenciados en Nutrición). Previamente se realizó una prueba piloto con el fin de validarlas y prever inconvenientes en su aplicación.
Se evaluaron las variables frecuencia semanal de desayuno (categorías: todos los días, a veces y nunca), factores vinculados a la frecuencia del desayuno: compañía (categorías: solo o con algún familiar) y preparación (categorías: solo o algún familiar); actividades realizadas durante el desayuno (categorías: mira televisión, hace la tarea, otras); causas por las que omite esta comida (categorías: falta de tiempo, falta de hambre, sensación de malestar); calidad del desayuno y calidad final contemplando la colación consumida a media mañana.

La variable calidad del desayuno se analizó según los criterios del estudio enKid, utilizado en numerosas investigaciones, ${ }^{1,13,17}$ que considera la incorporación de una porción de alimento de cada uno de los siguientes grupos: lácteos, cereales y fruta. Este criterio es coincidente con lo establecido por las Guías Alimentarias para la Población Argentina.${ }^{16}$ Se consideró "porción" la definida por el Código Alimentario Argentino en el Capítulo V. ${ }^{18}$ Para evaluar el tamaño de la porción se utilizaron elementos de medidas caseros (tazas, vasos, cucharas, y productos que se venden en fracciones como galletitas, panes en rodajas y potes de yogur).

Las categorías de la variable calidad de desayuno fueron:

- Buena calidad: incluye al menos una porción de cada uno de los tres grupos de alimentos considerados.

- Mejorable calidad: incluye una porción de alimento de dos grupos distintos.

- Insuficiente calidad: incluye una porción de alimento de un solo grupo.

- Mala calidad: incluye alimentos que no pertenecen a ninguno de estos tres grupos (infusiones, golosinas, etc.).

La colación se tomó como variable complementaria de la variable calidad de desayuno, considerando que la calidad mejora cuando a media mañana se ingiere el o los alimentos necesarios para obtener un desayuno de buena calidad.

Los resultados se expresaron para la totalidad de alumnos estudiados y por nivel escolar $\left(1^{\circ}\right.$ a $3^{\text {er }}$ grado, $4^{\circ}-5^{\circ}$ grado, $6^{\circ}-7^{\circ}$ grado). La información se procesó en forma sistematizada mediante el programa SPSS v17. Los resultados en las distintas categorías de las variables se expresaron en frecuencia relativa porcentual. Las asociaciones entre las variables frecuencia de desayuno y los factores considerados se evaluaron con la prueba de la $\chi^{2}$ y se calcularon los valores de odds ratio (OR). 


\section{RESULTADOS}

Los resultados corresponden a la totalidad de los alumnos encuestados, dado que ambas escuelas fueron consideradas como unidades homogéneas según los criterios de inclusión establecidos para este estudio. La muestra quedó constituida por 637 alumnos de ambos sexos: 282 escolares de $1^{\circ}$ a $3^{\text {er }}$ grado (con una edad promedio de $6,9 \pm 0,8$ años); 188 escolares de $4^{\circ}$ $5^{\circ}$ grado (con una edad promedio de $9,4 \pm 0,5$ años) y 167 escolares de $6^{\circ}-7^{\circ}$ grado (con una edad promedio de $11,4 \pm 0,5$ años).

La Figura 1 presenta los resultados de frecuencia de desayuno para la totalidad de los niños evaluados y por nivel escolar. Del total de niños encuestados, $75 \%$ manifestaron desayunar diariamente.

El análisis de los factores que podrían afectar la frecuencia semanal de desayuno mostró que no había asociación con el hecho de estar solo o acompañado por algún miembro de la familia a la hora de desayunar, para ninguno de los niveles escolares (valores $p>0,05$ ).

Para los alumnos de los grupos $1^{\circ}$ a $3^{\circ}$ y $4^{\circ}$ $5^{\circ}$ grado la frecuencia semanal de desayuno dependió de que algún familiar se lo preparara (valor $p=0,04$ ). Por cada niño que desayunaba diariamente, habiéndose preparado él mismo el desayuno, había 3 niños que lo hacían si se lo preparaba un familiar $(\mathrm{OR}=3)$. Para los más grandes $\left(6^{\circ}-7^{\circ}\right.$ grado), el hecho de desayunar diariamente o a veces no dependió de quién preparara el desayuno (valor $p=0,8$ ).

Se observó, en más de la mitad de los niños de todos los niveles escolares, que la principal actividad realizada durante el desayuno era

Figura 1. Frecuencia semanal de consumo de desayuno en los escolares

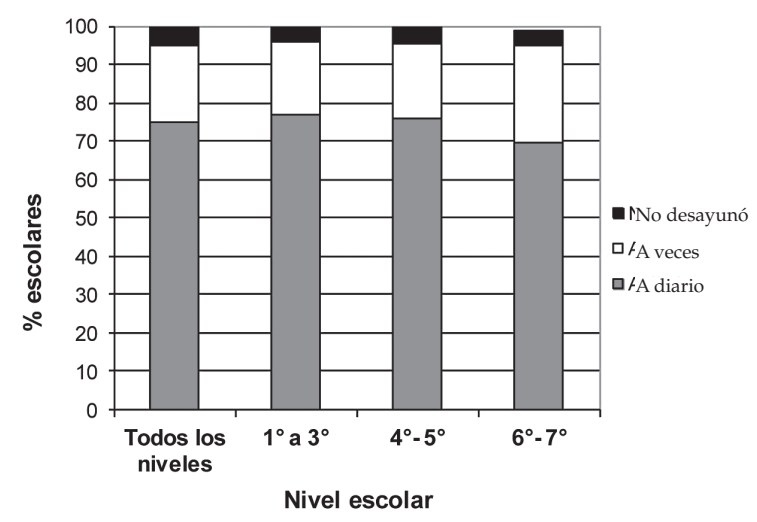

mirar televisión (Figura 2).

El principal motivo por el cual los alumnos de todos los niveles escolares manifestaron no desayunar o hacerlo a veces fue la falta de tiempo ( $40 \%$ del grupo $1^{\circ}$ a $5^{\circ}$ y $30 \%$ del grupo $\left.6^{\circ}-7^{\circ}\right)$. En segundo término, los alumnos de $1^{\circ}$ a $3^{\circ}$ y $4^{\circ}-5^{\circ}$ grado argumentaron no tener hambre. El $23 \%$ de los alumnos de $6^{\circ}-7^{\circ}$ grado manifestaron sentir malestar al desayunar; esta frecuencia fue mucho más baja en los otros dos niveles escolares (7\%).

El día de la encuesta solo 1,6\% de los niños consumieron un desayuno de buena calidad, $33,1 \%$ de mejorable calidad, $45,4 \%$ de insuficiente calidad, $8,9 \%$ de mala calidad, mientras un $11 \%$ no desayunó. Cuando se analizó la calidad del desayuno por niveles escolares, se observó un porcentaje similar de niños que no desayunaron en todos los grupos (Figura 3). La mayoría de los alumnos de $1^{\circ}$ a $3^{\text {er }}$ grado presentaron un desayuno de mejorable (41\%) e insuficiente calidad $(41 \%)$. En los alumnos de $4^{\circ}-5^{\circ}$ grado se apreció una disminución del desayuno de mejorable calidad $(30 \%)$ a expensas de un aumento del desayuno de insuficiente calidad (50\%). Esta misma tendencia se presentó en el grupo $6^{\circ}-7^{\circ}$ grado, en el que además aumentó la cantidad de niños que realizaron un desayuno de mala calidad (16\%).

Entre el $80 \%$ y el $90 \%$ de los niños consumieron una colación a media mañana. Tras la colación se observó en todos los niveles escolares, un ligero aumento en la proporción de niños que alcanzaron un desayuno de buena calidad, siendo significativo solamente para el grupo de $1^{\circ}$ a $3^{\text {er }}$ grado (valor $p=0,04$ ). Sin embargo, solo $6 \%$ de estos niños lograron un desayuno de buena calidad final (Tabla 1).

FIGURA 2. Actividades realizadas por los niños durante el desayuno

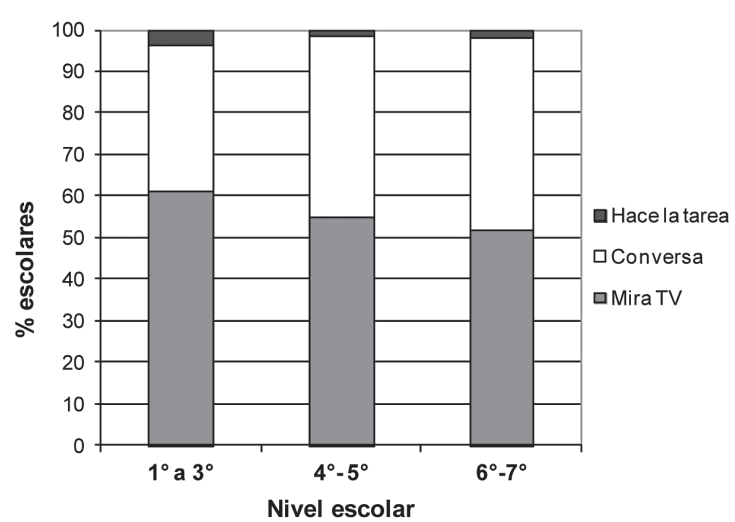




\section{DISCUSIÓN}

Los resultados muestran que la mayoría de los niños de $1^{\circ}$ a $7^{\circ}$ grado en las dos escuelas primarias consumen el desayuno diariamente $(75 \%)$, mientras que $5 \%$ nunca desayunan. Esta situación es semejante a la encontrada en un estudio realizado en niños españoles de entre 9 y 13 años. ${ }^{19}$ En nuestro estudio, no se observaron variaciones en la frecuencia del desayuno para los distintos niveles escolares, a diferencia de otra investigación, la cual mostró que los niños mayores (11 a 14 años) desayunan con menor frecuencia. ${ }^{20}$

El hábito de desayuno en los niños puede asociarse a diferentes factores. Se ha demostrado que la frecuencia de desayuno mejora cuando se lo toma en compañía. ${ }^{21}$ Sin embargo, en el presente estudio se observa que no existe dependencia entre dicha variable y el hecho de estar solo o acompañado por algún miembro de la familia, en ninguno de los niveles escolares. Una situación similar se encontró en otros estudios donde la mayoría de los niños desayunan solos. ${ }^{6,19}$

En relación con la preparación del desayuno, pudimos determinar que los niños de $1^{\circ}$ a $5^{\circ}$ grado desayunan con mayor frecuencia cuando se lo prepara un familiar. Es muy importante que la preparación del desayuno esté a cargo de un miembro de la familia. Los padres o tutores tienen la responsabilidad de proporcionar a los niños los alimentos apropiados para el desayuno..$^{22,23}$ Contrariamente, para los más grandes, el hecho de desayunar diariamente o a veces no depende de que se lo prepare algún miembro de la familia, por lo que ellos serían responsables de la elección de sus alimentos.

Figura 3. Calidad del desayuno según el nivel escolar

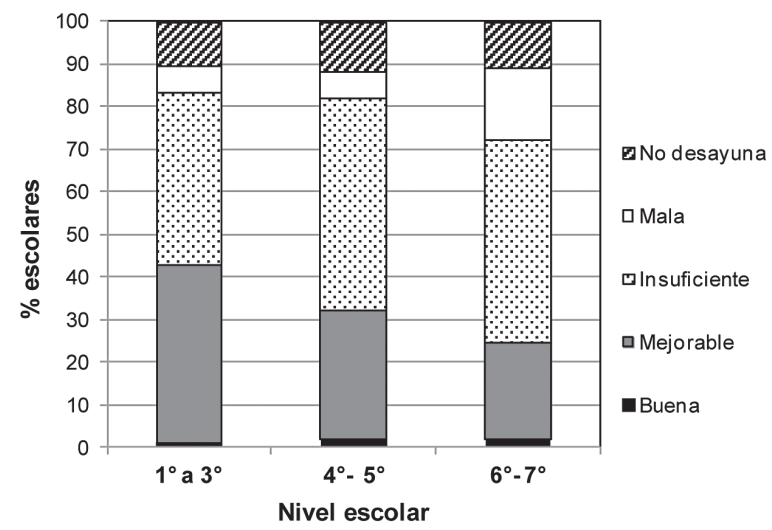

A la hora de desayunar, más del $50 \%$ de los niños de todas las edades miran televisión, situación similar a la observada en un estudio realizado en escolares españoles, donde $40 \%$ manifestaron mirar televisión durante el desayuno. ${ }^{24}$ Esto puede resultar un factor de distracción a la hora de ingerir los alimentos y puede restar tiempo para la realización del desayuno. Además, la publicidad televisiva determina, en gran medida, las preferencias de los niños por ciertos alimentos que, en general, no contribuyen a una alimentación saludable. ${ }^{25}$

En lo referente a las causas por las cuales no desayunan o lo hacen a veces, el principal motivo en todas las edades es la falta de tiempo. En segundo término, los niños de $1^{\circ}$ a $5^{\circ}$ grado argumentan no tener hambre. Resultados similares se obtuvieron en otros estudios, donde los principales motivos de omisión del desayuno fueron la falta de tiempo y no tener hambre a la mañana temprano. ${ }^{13,14}$ La falta de tiempo podría estar relacionada con la hora en que se van a dormir, la hora de inicio de las clases y no levantarse con el tiempo suficiente. Algunos autores demostraron que el tiempo empleado para desayunar está relacionado con el número de alimentos consumidos y esto condiciona la calidad del desayuno. ${ }^{21}$ En los alumnos de $6^{\circ}-7^{\circ}$ grado se suma otro factor, que es la sensación de malestar si desayunan antes de ir a la escuela.

En relación con la calidad del desayuno, del total de niños encuestados solo 1,6\% realizan un desayuno de buena calidad y la mitad consumen un desayuno de insuficiente calidad. Valores similares resultan para esta variable en los distintos niveles escolares. En un estudio realizado en niños del Gran Buenos Aires, donde se analizó la calidad del desayuno solo desde el punto de vista cualitativo, se encontró que solamente $5 \%$ realizaban un desayuno de buena calidad. ${ }^{13}$ Dos estudios efectuado en España en niños de 9 a 13 años hallaron que entre $8 \%$ y $10 \%$ incluyeron lácteos, cereales y frutas. ${ }^{26,19}$

TABLA 1. Escolares que consumen un desayuno de buena calidad antes y después de la colación

\begin{tabular}{|c|c|c|c|}
\hline & $1^{\circ}$ a $3^{\circ}$ grado & $4^{\circ}$ a $5^{\circ}$ grado & $6^{\circ}$ a $7^{\circ}$ grado \\
\hline $\begin{array}{l}\text { Desayuno de } \\
\text { buena calidad }\end{array}$ & $n=282$ & $n=188$ & $n=167$ \\
\hline A primera hora del día & $1,1 \%$ & $2 \%$ & $1,1 \%$ \\
\hline Después de la colación & $6 \% *$ & $4,5 \%$ & $1,7 \%$ \\
\hline
\end{tabular}


En otro trabajo realizado en España con niños inmigrantes de edad promedio de 11 años, los resultados fueron muy variables según el país de procedencia, de modo que el consumo de desayuno de buena calidad osciló entre 11,5\% y $51,5 \% .{ }^{21}$ Como se observa, el consumo de desayuno de buena calidad es muy variable según la población infantil estudiada. Hay que considerar que la mayoría de los niños encuestados en este estudio desayunan solos y quizá son responsables de elegir los alimentos que consumen en esta comida, lo que podría condicionar la calidad del desayuno. ${ }^{24}$

$\mathrm{Al}$ analizar la calidad del desayuno en función del nivel escolar, se observa que la mayoría de los alumnos de $1^{\circ}$ a $3^{\text {er }}$ grado consumen un desayuno de mejorable o insuficiente calidad. En los de $4^{\circ}$ y $5^{\circ}$ grado se observa un aumento del desayuno de insuficiente calidad. Esta misma tendencia se aprecia en $6^{\circ}$ y $7^{\circ}$ grado, pero en este grupo se incrementa la cantidad de niños que realizan un desayuno de mala calidad. Esto coincide con trabajos previos que sostienen que a medida que aumenta la edad tienden a consumir desayunos más ligeros. ${ }^{27}$ Se han encontrado correlaciones inversas entre la edad y los tipos de alimentos consumidos en el desayuno; es decir, los escolares de mayor edad incluyen menor variedad de alimentos. ${ }^{6}$ En otro estudio se detectó una mayor proporción de niños de 12 a 13 años $(16 \%)$ que realizaban un desayuno de buena calidad. ${ }^{1}$ A pesar de las variaciones observadas en los distintos trabajos, siempre son muy pocos los niños que consumen un desayuno de buena calidad.

Entre $80 \%$ y $90 \%$ de los niños consumen un alimento a media mañana. Sin embargo, la mayoría de los encuestados de ambas escuelas no logran completar la calidad del desayuno con la colación. Esta situación probablemente puede atribuirse a la falta de conocimiento de los niños en lo que respecta a los alimentos que deben incluir en el desayuno y la colación. Además, los niños disponen de dinero y es probable que reciban escasa orientación de los adultos en la elección de los alimentos. A esto debe sumarse que los kioscos escolares ofrecen una variedad de productos muy apetecibles que no contribuyen a mantener una alimentación saludable. Por su parte, Fernández San Juan y cols., observaron que los niños que acuden a la escuela sin desayunar habitualmente tienen dinero para comprar alimentos, los que suelen ser de baja calidad nutricional. ${ }^{28}$
Cabe destacar que, en el presente estudio, la evaluación de la calidad del desayuno se realizó considerando la cantidad de alimento ingerida, apreciación no tenida en cuenta en otros estudios. Esto permitió evaluar el desayuno según las recomendaciones de las Guías Alimentarias para la Población Argentina. Una limitación natural en este tipo de trabajo es que la información es referida por un participante, por lo cual no se puede "asegurar" que cada niño haya respondido con absoluta veracidad y exactitud.

Los resultados del presente estudio no permiten hacer inferencias a la población escolar primaria, ya que la muestra solo es representativa de escuelas con características semejantes a las consideradas en este estudio. A pesar de esta limitación, es válido aconsejar aplicar acciones de educación alimentaria nutricional para los alumnos de nivel primario, con el objeto de generar tempranamente un buen hábito de desayuno que perdure a lo largo de la vida. Por otro lado, el alcance de estas acciones debería incluir a la familia, ya que es uno de los determinantes importantes en las preferencias alimentarias de la población infantil.

\section{CONCLUSIONES}

E1 $75 \%$ de los escolares desayunan diariamente; solo 1,6\% consumen un desayuno de buena calidad. El 50\% de los niños consumen solo una porción de alimento de los recomendados a primera hora de la mañana y la colación no resulta apropiada para completar la calidad final del desayuno.

Los factores asociados a la omisión del desayuno son la falta de tiempo (36\%), la falta de hambre $(21 \%)$, sentirse mal al momento de desayunar ( $23 \%$ de los alumnos de $6^{\circ}-7^{\circ}$ grado). Para los alumnos de $1^{\circ}$ a $5^{\circ}$ grado, la frecuencia semanal de desayuno depende de que algún familiar se lo prepare (valor $p=0,04$ ).

\section{Agradecimientos}

A Juan Villafañe, presidente de la Federación de Asociaciones de Cooperadoras Escolares del Departamento La Capital de Santa Fe, por preocuparse y ocuparse de la alimentación infantil. A los directores, maestros y niños que participaron con entusiasmo en este proyecto.

\section{BIBLIOGRAFÍA}

1. Herrero Lozano R, Fillat Ballesteros JC. Estudio sobre el desayuno y el rendimiento escolar en un grupo de adolescentes. Nutr Hosp 2006;21(3):346-52.

2. JofréJM,Arenas MC,Azpiroz R, DeBertoliMA.Importancia 
del desayuno en el estado nutricional y el procesamiento de la información en escolares. Universidad de Psicología de Bogotá 2007;6(2):371-82.

3. Pivik RT, Tennal KB, Chapman SD, Gu Y. Eating breakfast enhances the efficiency of neural networks engaged during mental arithmetic in school-aged children. Physiology $\mathcal{E}$ Behaviour 2012;106:548-55.

4. Wesnes K, Pincock C, Scholey A. Breakfast is associated with enhanced cognitive function in schoolchildren. An internet based study. Appetite 2012;59:646-49.

5. Nicklas TA, Bao W, Webber LS, Berenson GS. Breakfast consumption affects adequacy of total daily intake in children. J Am Diet Assoc 1993;93(8):886-91.

6. Ortega RM, González-Rodríguez LG, Jiménez Ortega AI, Perea Sánchez JM, et al. The importance of breakfast in meeting daily recommended calcium intake in a group of schoolchildren. Am J Coll Nutr 1998;17:19-24.

7. Sampson AE, DixitS, Meyers AF, Houser R. The nutritional impact of breakfast consumption on the diets of inner-city African-American elementary school children. J Nat Med Assoc 1995;87(3):195-202.

8. Siega-Riz AM, Popkin BM, Carson T. Trends in breakfast consumption for children in the United States from 19651991. J Am Clin Nutr 1998;67:748-56.

9. Sjôberg A, Hallberg L, Hôglund D, Hulthén L. Meal pattern, food choice, nutrient intake and lifestyle factors in the Gôteborg Adolescence Study. European J Clin Nutr 2003;57:1569-78.

10. Rampersaud GC, Pereira MA, Girard BL, Adams J, et al. Breakfast habits, nutritional status, body weight, and academic performance in children and adolescents. J Am Diet Assoc 2005;105(5):743-60.

11. Deshmukh-Taskar PR, Nicklas TA, O’Neil CE, Keast DR, et al. The Relationship of Breakfast Skipping and Type of Breakfast Consumption with Nutrient Intake and Weight Status in Children and Adolescents: The National Health and Nutrition Examination Survey 1999-2006. J Am Diet Assoc 2010;110:869-78.

12. Serra Majem L, Ribas Barba L, Aranceta Bartrina J, Pérez Rodrigo C, et al. Obesidad infantil y juvenil en España. Resultados del Estudio en Kid (1998-2000). Medicina Clínica 2003;121(19):725-32.

13. Angeleri MA, González I, Ghioldi MM, Petrelli L. Hábito de consumo del desayuno y calidad nutricional del mismo en niños y adolescentes de la zona norte del gran Buenos Aires. DIAETA 2007;25(116):7-13.

14. Reddan J, Wahlstrom K, Reicks M. Children's perceived benefits and barriers in relation to eating breakfast in schools with or without Universal School Breakfast. J Nutr Educ Behav 2002;34:47-52.

15. O'Sullivan TA, Robinson M, Kendall GE, Miller M, et al. A good-quality breakfast is associated with better mental health in adolescence. Public Health Nutr 2009;12(2):249-58.
16. Asociación Argentina de Dietistas y Nutricionistas Dietistas (AADyND). Guías Alimentarias para la Población Argentina. Consejos para una alimentación saludable. Argentina, 2000. [Acceso: 5 de septiembre de 2012]. Disponible en: http:/ /www.aadynd.org.ar/guias_ consejos.php.

17. Fernández Morales I, Aguilar Vilas M, C. J, Mateos Vega C y Martínez M. Relación entre la calidad del desayuno y el rendimiento académico en adolescentes de Guadalajara (Castilla-La Mancha). Nutr Hosp 2008;23(4):383-7.

18. Código Alimentario Argentino. Capítulo V: Normas para la rotulación y publicidad de los alimentos. Resolución GMC n 47/03 - "Reglamento Técnico Mercosur de porciones de alimentos envasados a los fines del rotulado nutricional". Última actualización: 2/2013. [Acceso: 20 de abril de 2013]. Disponible en: http://www.anmat.gov.ar/alimentos/ codigoa/Capitulo_V.pdf].

19. Amat Huerta MA, Anuncibay Sánchez V, Soto Volante J, Alonso Nicolás J, et al. Estudio descriptivo sobre hábitos alimentarios en el desayuno y almuerzo de los preadolescentes de Viladecans (Barcelona). Nure Investigación 2006;23:

20. Utter J,Scragg R,Ni Murchu G,SchaafD. At-homebreakfast consumption among New Zealand children: associations with body mass index and related nutrition behaviours. J Am Diet Assoc 2007;107:570-6.

21. Menal-Puey S, Fajó-Pascual M, Marques-Lopes I. Estudio descriptivo del desayuno en una población infantil inmigrante escolarizada, 2007-2010. Rev Esp Nutr Hum Diet 2011;15(4):177-83.

22. Affenito SG. Breakfast: a missed opportunity. J Am Diet Assoc 2007;107(4):565-9.

23. Pearson N, BiddleS, Gorely T. Family correlates of breakfast consumption among children and adolescents. A systematic review. Appetite 2009;52:1-7.

24. Castells Cuixart M, Capdevila Prim C, Girbau Solà T, RodríguezCabC. Estudio del comportamiento alimentario en escolares de 11 a 13 años de Barcelona. Nutr Hosp 2006;21(4):517-32.

25. Patrick HP, Nicklas TA. A Review of Family and Social Determinants of Children's Eating Patterns and Diet Quality. J Am College of Nutr 2005;24(2):83-92.

26. Ortega RM, Requejo AM, Redondo R, et al. Breakfast habits of different groups of Spanish schoolchildren. J Hum Nutr Dietet 1996,9:33-41.

27. Ruxton $\mathrm{CH}$,Kirk TR. Breakfast: a review of associations with measures of dietary intake, physiology and biochemistry. British Journal of Nutrition 1997;78:199-213.

28. Fernández San Juan PM. Dietary habits and nutritional status of school aged children in Spain. Nutr Hosp 2006;21(3):374-8. 


\section{Anexo 1}

Escuela:

Nombre:

Peso:
Grado:

Edad:

Talla:

Sexo:

1) ¿Desayunas? $\quad \square$ Todos los días (6 o 7 días)

A veces (1-5 días) $\square$ Nunca

2) ¿Por qué no desayunas todos los días?

$\square$ No tengo tiempo

$\square$ No tengo hambre

$\square$ Me hace mal

Otros (especificar)

3) ¿Quién prepara el desayuno?

$$
\square \text { Yo solo }
$$

4) ¿Con quién sueles desayunar?

$\square$ Solo $\quad \square$ Con mi familia

5) ¿Qué haces mientras desayunas?

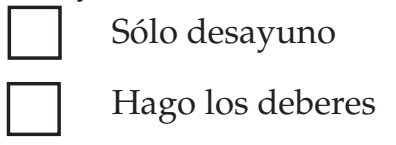

Algún familiar

Con otras personas (especificar)

6) Hoy, antes de venir a la escuela, ¿qué comiste y bebiste?

Detallar todos los alimentos y bebidas con sus respectivas porciones

7) Durante la mañana, ¿vas a comer o tomar algo?

Detallar los alimentos consumidos en la colación con sus respectivas porciones 